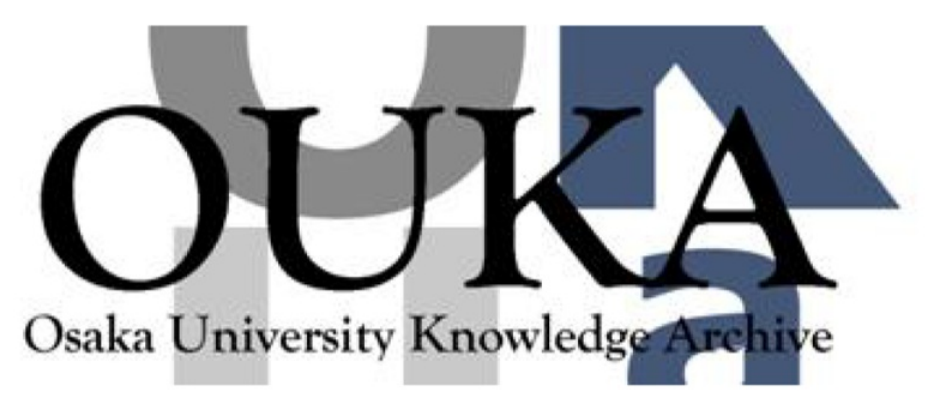

\begin{tabular}{|c|l|}
\hline Title & $\begin{array}{l}\text { Reagent-free crosslinking of aqueous ge lat in } \\
\text { manufacture and characteristics of ge lat in ge ls } \\
\text { irradiated with gamma-ray and electron beam }\end{array}$ \\
\hline Author(s) & $\begin{array}{l}\text { Furusawa, Kazuya; Mori, Yasutaka; Yoshi i, Fumio } \\
\text { et al. }\end{array}$ \\
\hline Citation & $\begin{array}{l}\text { Journal of Biomaterials Science, Polymer } \\
\text { Edition. 14(11) p.1197-p. 1208 }\end{array}$ \\
\hline Issue Date & $2003-11$ \\
\hline oaire:version & AM \\
\hline URL & https://hdl. handle.net/11094/25935 \\
\hline rights & \\
\hline Note & \\
\hline
\end{tabular}

Osaka University Knowledge Archive : OUKA

https://ir. Library. osaka-u. ac. jp/

Osaka University 


\section{Reagent-free crosslinking of aqueous gelatin: manufacture and characteristics of gelatin gels irradiated with gamma-ray and electron beam}

KEN TERAO $^{1, *}$, NAOTSUGU NAGASAWA ${ }^{2}$, HIROKAZU NISHIDA ${ }^{1}$, KAZUYA FURUSAWA $^{1}$, YASUTAKA MORI ${ }^{1}$, FUMIO YOSHII $^{2}$ and TOSHIAKI DOBASHI ${ }^{1}$

${ }^{1}$ Department of Biological and Chemical Engineering, Faculty of Engineering, Gunma University, Kiryu, Gunma 376-8515, Japan.

${ }^{2}$ Takasaki Radiation Research Establishment, Japan Atomic Energy Research Institute, Watanuki-machi, Takasaki, Gunma 370-1292, Japan.

Short title: Radiation crosslinking of aqueous gelatin

* To whom correspondence should be addressed. E-mail: terao@bce.gunma-u.ac.jp 


\begin{abstract}
In order to obtain a gelatin hydrogel crosslinked by a reagent-free method, gamma-ray and electron beam radiation was applied to porcine, bovine and fish gelatin gels and the products were characterized by measuring the gel fraction, the swelling ratio and the enzymatic degradability. As increasing the radiation dose, the gel fraction increased and both the swelling ratio and the enzymatic degradability decreased. The transition temperature from gel to sol of the hydrogel containing more than $5 \%$ mammal gelatins increased up to more than $90^{\circ} \mathrm{C}$ when gamma-ray or electron beam were irradiated by more than $10 \mathrm{kGy}$. The results show that the degree of crosslinking of irradiated gelatin hydrogels increases with increasing irradiation dose and with decreasing concentration. It is suggested that the radiation crosslinking occurs around the physical crosslinking point or multiple helix structure of gelatin gel.
\end{abstract}

Key words: hydrogels; gelatin; biodegradability; radiation; electron beam; crosslinking; proteinase; reagent-free. 


\section{INTRODUCTION}

Gelatin, whose non-dilute solutions exhibit a reversible (physical) sol-gel transition between 25 $40^{\circ} \mathrm{C}$, is one of the most useful biopolymers for clinical medicine, food processing and so on, because the biosafety of gelatin has been confirmed by food and clinical applications for a long period [1]. It is, however, difficult to use the gelatin gel without processing as a carrier of the drug delivery system because the sol-gel transition temperature is too close to the mammal's body temperature. Recently, Tabata et al. found that the gelatin hydrogels crosslinked with glutaraldehyde were useful as a carrier of a drug delivery system [2]. Furthermore, they found that the desorption of some growth factors from the hydrogel could be controlled through the biodegradability of the hydrogel [3, 4]. It should be noticed, however, that the hydrogel thus obtained is not suitable for every clinical medicine because glutaraldehyde has significant toxicity and oncogenicity $[5,6]$. Therefore, it has been desirable to develop methods manufacturing gelatin hydrogels by a reagent-free method. Very recently, some groups found out that thin gelatin films could be crosslinked with UV light and the gel film thus obtained is suitable for medical use [7, 8]. A disadvantage of this method is that only thin films could be applied because UV light hardly penetrates into thick gelatin gels and the degree of crosslinking of them is much smaller than those made using formaldehyde [8(a)].

Radiation is one of convenient tools to modify polymers, e.g. crosslinking and degradation, without any chemical reagents. Whether a polymer can be crosslinked (crosslinking polymer) or degraded (scission polymer) depends on the monomer unit of the polymer. For gelatin, it has been considered to be a scission polymer, because when a powder of gelatin was irradiated, the solution viscosity of the gelatin decreased with increasing irradiation dose [9]. On the other hand, although cellulose derivatives were classified as a scission polymer [10], sodium 
carboxymethylcellulose $[11,12]$ and hydroxypropylcellulose [13] were found to be crosslinked by gamma-ray or electron beam when their concentrated solutions were irradiated. Moreover, it was found that the degree of crosslinking and the enzymatic degradability could be controlled by the condition of irradiation [12 - 14]. Therefore, we tried to perform an irradiation for concentrated aqueous gelatin. At the first stage of this study, we have found that the melting temperature of the hydrogel containing more than 5\% mammal gelatins increased up to more than $90^{\circ} \mathrm{C}$ when gamma ray generated from a ${ }^{60} \mathrm{Co}$ source at a dose rate of $10 \mathrm{kGy} / \mathrm{h}$ or electron beam irradiated more than $10 \mathrm{kGy}$ whereas the hydrogel irradiated less than $5 \mathrm{kGy}$ melted less than $30^{\circ} \mathrm{C}$. It was expected that the hydrogel thus obtained is more suitable than that crosslinked with glutaraldehyde because no chemical reagent is needed in the preparation process, and the hydrogels are completely homogeneous and easily processed, which could not be obtained by using any crosslinking reagents. The present paper reports on the preparation method and the characteristics of the gelatin hydrogels by radiation crosslinking.

\section{METHODS}

\section{Materials}

Three gelatin samples from bovine skin (type A, treated with an acid), porcine skin (type B, treated with a base), and cold water fish skin (type A) were purchased from Sigma Co. and used without further purification. These gelatin samples were dissolved at $45^{\circ} \mathrm{C}$ in pure water (for the experiments of gel fraction and swelling ratio) or a phosphate buffer (for enzymatic degradation) with the mass concentration in the range from 0.01 to $0.30 \mathrm{~g} \mathrm{~cm}^{-3}$. Here, we note that the biodegradability of the hydrogel made with the use of pure water was essentially the same as that in the buffer solution. The gelatin solutions were irradiated with electron beam 
from a $3 \mathrm{MeV}$ accelerator. The dose rate, the irradiated dose per passing under the light source, was $1 \mathrm{kGy}$ pass $^{-1}$ as long as it was not mentioned below. Generally, the temperature of the sample rises with increasing dose rate. For the irradiation with electron beam, gel sheets whose thickness $\delta$ was $1.0 \mathrm{~mm}$ and $0.5 \mathrm{~mm}$ was prepared using a cold-press equipment and heat-sealed in a poly(vinylidene chloride) bag after removal of the air by a vacuum machine in order to shut out air during the irradiation. The irradiation was made at a room temperature around $20^{\circ} \mathrm{C}$.

Gel fraction and swelling ratio of hydrogel

The gel fraction $G_{\mathrm{f}}$ defined as the weight ratio of the dried insoluble portion to the dried original gel was determined from the gravimetry under the following procedures. The gel was dried at $30^{\circ} \mathrm{C}$ under atmospheric pressure for $24 \mathrm{~h}$ and then in vacuum for $24 \mathrm{~h} . \quad$ After the weight of the dried original gel was measured, it was soaked in deionized water at $50^{\circ} \mathrm{C}$ for 2 days in order to elute the soluble part to water. The insoluble portion was separated with a stainless steel net (150 mesh) and was weighed after drying. The swelling ratio $S_{\mathrm{r}}$ was determined as the weight ratio of the swollen gel to the dried original gel. The weight of the swollen gel was measured as follows; the original dried gel was soaked in deionized water at room temperature for 2 days to obtain the hydrogel, and the portion filtered by the net and lightly blotted out was weighed.

\section{Biodegradation}

An enzymatic biodegradation measurement was carried out for the irradiated gel sheets of gelatin in an $0.02 \mathrm{M}$ sodium bicarbonate / $0.001 \mathrm{M}$ calcium chloride buffer solutions with or without proteinase of $0.01 \mathrm{~g} \mathrm{~cm}^{-3}$ as an enzyme at $40^{\circ} \mathrm{C}$. The activity of the proteinase (Tokyo Kasei) 
from bacillus subtilis was stated to be about 89,000 units $\mathrm{g}^{-1}$ with using casein as a substrate according to the data sheet issued by the corporation. Each gel sheet was cut into a square with the side being $20 \mathrm{~mm}$, soaked in a $30 \mathrm{~cm}^{3}$ buffer solution and gently stirred. UV absorbance of the buffer solution at $275 \mathrm{~nm}$ wavelength resulting from the aromatic amino acids consisting of the dissolved gelatin molecule was measured at appropriate time intervals. Thus, the time course of the concentration of gelatin dissolved in the proteinase solution could be estimated [15]. For the experimental condition when the gelatin concentration is small and the irradiation dose is very large, however, shrinking of the gel sheet and a syneresis (a phenomena that buffer in the hydrogel oozed out) was observed, the degradation rate is very small and the time course of UV absorbance is too complicated to determine the time for complete biodegradation. The same measurement was carried out for a chemically crosslinked gelatin sheet prepared for comparison between two types of crosslinking; an appropriate amount of gelatin was dissolved in water, an appropriate amount of aqueous 5\% glutaraldehyde (Wako) was poured into the gelatin solution and stirred vigorously. Finally we obtained a hydrogel containing $0.1 \mathrm{~g} \mathrm{~cm}^{-3}$ gelatin and $10 \mathrm{mM}$ glutaraldehyde. Enzymatic degradation experiments were also carried out for a gelatin sheet made from gelatin in pure water (containing no phosphate salts) in order to investigate the difference of solvents. We observed no significant difference between these two systems.

\section{Circular dichroism}

To investigate the helical contents of the difference of the source of gelatin, circular dichroism measurements for two gelatin samples from porcine and bovine in the phosphate buffer at $\mathrm{pH} 7.0$ were made on a JASCO J700 circular dichroism spectropolarimeter using a quartz cell of $0.1 \mathrm{~cm}$ path length at wavelength of the incident light between 190 and $240 \mathrm{~nm}$. The solutions whose 
concentration was $1 \times 10^{-4} \mathrm{~g} \mathrm{~cm}^{-3}$ were prepared for this study.

\section{RESULTS AND DISCUSSION}

\section{Gel fraction and swelling ratio}

Figure 1(a) shows the dose dependence of $G_{\mathrm{f}}$ for aqueous porcine skin irradiated with electron beam. The both experimental errors for $G_{\mathrm{f}}$ and $S_{\mathrm{r}}$ were estimated to be about $\pm 10 \%$ from the difference of the data obtained from the same conditions. $G_{\mathrm{f}}$ for the mass concentration $c$ of $0.05,0.10,0.20$, and $0.30 \mathrm{~g} \mathrm{~cm}^{-3}$ increase rapidly in the range between 5 and $20 \mathrm{kGy}$ and reach a constant, whereas those for 0.02 and $0.01 \mathrm{~g} \mathrm{~cm}^{-3}$ are almost zero. This result suggests that the concentration of $0.02-0.05 \mathrm{~g} \mathrm{~cm}^{-3}$ is the boundary concentration to crosslink the gelatin gel by the electron beam at the radiation more than $5 \mathrm{kGy}$. The hydrogels with the concentration being more than $0.05 \mathrm{~g} \mathrm{~cm}^{-3}$ irradiated with $30 \mathrm{kGy}$ gamma-ray or electron beam did not melt even at $90^{\circ} \mathrm{C}$, whereas the hydrogels irradiated with 0 and $5 \mathrm{kGy}$ melted around $30^{\circ} \mathrm{C}$. The concentration dependence of $G_{\mathrm{f}}$ for the same system is illustrated in Figure 1(b). A peak is observed around $0.1 \mathrm{~g} \mathrm{~cm}^{-3}$ and it becomes indistinct with increasing dose. This result shows that the chemical crosslinking behavior is correlated with the structure of the physical gel and presumably the number of chemical crosslinking points in the hydrogel becomes at the maximum around this concentration. The increase in $G_{\mathrm{f}}$ was also observed for aqueous bovine gelatin. On the other hand, the gel fraction of the irradiated hydrogels from cold water fish was almost zero for all conditions investigated (not shown in Figure 1). These results are very different from those obtained for the chemically crosslinked gelatin, i.e., aqueous gelatins from all species can be easily crosslinked by glutaraldehyde. 
The aqueous gelatins with the concentration being more than $0.05 \mathrm{~g} \mathrm{~cm}^{-3}$ are at the (physical) gel state in the present condition of the irradiation at room temperature. If we assume that the radiation crosslinking occurs at and/or near the physically crosslinking point (or multiple helical structure), the difference of gelatins between the fish and the mammals is consistent with the experimental fact that the helix content and the storage elastic modulus of the fish gelatin solution were much smaller than those of mammals $[16,17]$. Figure 2 shows the dose dependence of the swelling ratio $S_{\mathrm{r}}$ for the porcine skin gelatin. $S_{\mathrm{r}}$ decreases with increasing dose in the present range of concentration. This result suggests that the crosslinking degree increases with increasing irradiation dose, even after the gel fraction has reached a constant value. This trend is also observed for sodium carboxymethylcellulose investigated previously [12].

\section{Enzymatic degradation behavior}

Figure 3 illustrates two examples of degradation behavior of an irradiated gelatin gel in a buffer solution with or without enzyme. The degradation of the gel sheet should be proportional to the surface area, then the UV absorbance curve for the thin sheet must be a linear function of time until the sheet is completely degraded. Indeed, as shown for a typical experimental condition in panel (a), the UV absorbance of the buffer solution containing the enzyme increases almost linearly and becomes at an even value of $A_{\infty}$ after the time $\tau$ when the gel disappeared completely from the solution, whereas the UV absorbance from the buffer solution without enzyme increases very slowly. The slow increase observed in the latter solution reflects the desorption of the soluble part of the hydrogel. Thus, the rapid increase observed in the former solution is mainly attributed to the enzymatic degradation by the proteinase. For the experimental condition of low 
gelatin concentration and very large dose, we could not estimate $\tau$ because of the complicated behavior of the time course of UV absorbance, as shown in panel (b). $\tau$ determined from the UV absorbance was summarized in Table 1. It is shown from the fifth and sixth columns that the dose rate dependence was negligible within the experimental error. The effects of the irradiation dose for the $0.2 \mathrm{~g} \mathrm{~cm}^{-3}$ gelatin gel and the gelatin concentration at a fixed irradiation dose, $60 \mathrm{kGy}$, on $\mathrm{t}$ were shown in Figure 4(a) and (b), respectively. Each absorbance increases roughly linearly and reaches the respective equilibrium value without exceptions. $\tau$ increases with increasing dose and with decreasing the concentration of gelatin. These tendencies are consistent with those for the gel fraction and the swelling ratio.

$\tau$ is linearly proportional to irradiation dose in double logalithmic plots for the samples with different gelatin concentrations for the thickness of the gel sheet being $1 \mathrm{~mm}$, as shown in Figure 5. The lines have a slope of 1.2. The strong dependence of $\tau$ against dose shows that the biodegradability of gelatin gel crosslinked by radiation can be adjusted in a very wide range only by choosing an appropriate dose. Especially, the radiation crosslinking has an advantage for manufacturing gelatin hydrogels which typically weakly biodegradable and lack uniformity. Uniform hydrogels are difficult to manufacture by chemical crosslinking by glutaraldehyde because it is hard to make a uniform mixture of aqueous gelatin in the presence of a large volume of glutaraldehyde. As shown in Table 1, $\tau$ for bovine skin are much smaller than those for porcine skin at the same concentration and dose. This is consistent with the circular dichroism results that the helical contents of the gelatin solution from bovine skin was significantly smaller than those for porcine skin at the same temperature between 20 and $30^{\circ} \mathrm{C}$, as shown in Figure 6 because the helical contents are usually correlated positively with the number of the physical 
crosslinking points of the hydrogel.

The gel thickness dependence of biodegradability is seen from Table $1 \tau$ of the hydrogel for $c=$ $0.1 \mathrm{~g} \mathrm{~cm}^{-3}$ and $\delta=0.5 \mathrm{~mm}$ at $30 \mathrm{kGy}$ was about half as large as that for $\delta=1 \mathrm{~mm}$ at the same dose and $c ; \tau$ is in proportional to $\delta$. This result suggests that the gel was degraded from the surface. An exception is for the sample with the irradiation dose of $120 \mathrm{kGy}$; the value of $\tau$ for $120 \mathrm{kGy}$ and $\delta=1.0 \mathrm{~mm}$ is only about 1.4 times larger than that of $\delta=0.5 \mathrm{~mm}$. It might be attributed to the diffusion of the enzyme into the gel sheet irradiated with $120 \mathrm{kGy}$ in which the degree of crosslinking is quite high and the degradation velocity is very slow.

\section{CONCLUSIONS}

Aqueous gelatins from porcine skin crosslinked by gamma-ray and electron beam were characterized by the gel fraction, the swelling ratio, and the enzymatic biodegradability as a function of the irradiation dose and the concentration of gelatin. The results suggested that the degree of crosslinking increased monotonously with increasing dose and decreased with increasing concentration of gelatin. The latter result could be explained by assuming that the crosslinking reaction occurs at or near the multiple crosslinking structure (helical content of the gelatin) in the gelatin gel. This assumption is consistent with the different behaviors for gelatins from bovine skin and cold water fish skin. It is expected that the biodegradability of the gelatin solutions irradiated by electron beam can be controlled in a much broader range than that manufactured with glutaraldehyde. 


\section{Acknowledgement}

We thank Dr. Miyoko Kubo of Kawasaki Medical School, who drew their attention to crosslinked gelatin.

\section{REFFERENCES}

1. D. Zekorn, Bibl Haematol. 33, 131 (1969).

2. Y. Tabata and Y. Ikada, Adv. Drug Delivery Rev. 31, 287 (1998) and references therein.

3. Y. Tabata, A. Nagano, M. Muniruzzaman and Y. Ikada, Biomaterials 19, 1781 (1998).

4. M. Yamamoto, Y. Ikada and Y. Tabata, J. Biomater. Sci. Polymer Edn. 12, 77 (2001).

5. B. Ballantyne and S. L. Jordan, J. Appl. Toxicol. 21, 131 (2001) and references therein.

6. J. P. Van Miller, S. J. Hermansky, P. E. Losco and B. Ballantyne, Toxicology 175, 177 (2002).

7. A. Sugiyama, T. Sugie and H. Yanagawa, Jpn. Kokai Tokkyo Koho H11-47258, Feb, 23 (1999).

8. (a) S. Matsuda, N. Se, H. Iwata and Y. Ikada, Biomaterials 23, 2901 (2002). (b) N. Se, S.

Matsuda, H. Iwata and Y. Ikada, Jpn. Kokai Tokkyo Koho 2000-34750, Feb, 8 (2000).

9. F. F. Vieira and N. L. D. Mastro, Radiation Phys. Chem. 63, 331 (2002).

10. Y. Tabata, Y. Ito and S. Tagawa (Eds.) CRC Handbook of Radiation Chemistry. CRC Press, Boston (1991).

11. B. Fei, R. A. Wach, H. Mitomo, F. Yoshii and T. Kume, J. Appl. Polym. Sci. 78, 278 (2000).

12. R. A. Wach, H. Mitomo, F. Yoshii and T. Kume, J. Appl. Polym. Sci. 81, 3030 (2001).

13. R. A. Wach, H. Mitomo, F. Yoshii and T. Kume, Macromol. Mater. Eng. 287, 285 (2002). 
14. K. Terao, H. Nishida, S. Kondo, T. Dobashi, F. Yoshii, T. Hashimoto and S. Yagihara, Trans. Mater. Res. Soc. Jpn. 27, 593 (2002).

15. P. Dalev, E. Vassileva, J. E. Mark and S. Fakirov, Biotechnol. Tech. 12, 889 (1998).

16. C. Joly-Duhamel, D. Hellio and M. Djabourov, Langmuir 18, 7208 (2002).

17. C. Joly-Duhamel, D. Hellio, A. Ajdari and M. Djabourov, Langmuir 18, 7158 (2002). 
Table 1. Decomposition time $\tau$ for the irradiated gelatin gel

\begin{tabular}{|c|c|c|c|c|c|c|c|}
\hline $\mathrm{c} / \mathrm{g} \mathrm{cm}^{-3}$ & $0.20^{* 1}$ & $0.10^{* 1}$ & $0.050^{* 1}$ & $0.10^{* 1}$ & $0.10^{* 1}$ & $0.20^{* 2}$ & $0.10^{* 2}$ \\
\hline$\delta / \mathrm{mm}$ & 1.0 & 1.0 & 1.0 & 0.5 & 0.5 & 1.0 & 1.0 \\
\hline Dose Rate / kGy pass ${ }^{-1}$ & 1.0 & 1.0 & 1.0 & 1.0 & 10 & 1.0 & 1.0 \\
\hline Dose / kGy & \multicolumn{7}{|c|}{ Decomposition time $\tau / 10^{3} \mathrm{sec}$} \\
\hline 0 & - & $2.1^{* 3}$ & - & - & - & - & - \\
\hline 30 & 3.2 & 4.8 & 6.9 & 2.2 & 2.3 & 2.3 & 2.9 \\
\hline 60 & 7.7 & 11 & 18 & - & - & - & - \\
\hline 120 & 16 & 30 & - & 21 & 21 & - & - \\
\hline
\end{tabular}

\footnotetext{
${ }^{* 1}$ Gelatin from porcine skin (type A).

${ }^{* 2}$ Gelatin from bovine skin (type B).

${ }^{* 3}$ Crosslinked by glutaraldehyde.
} 

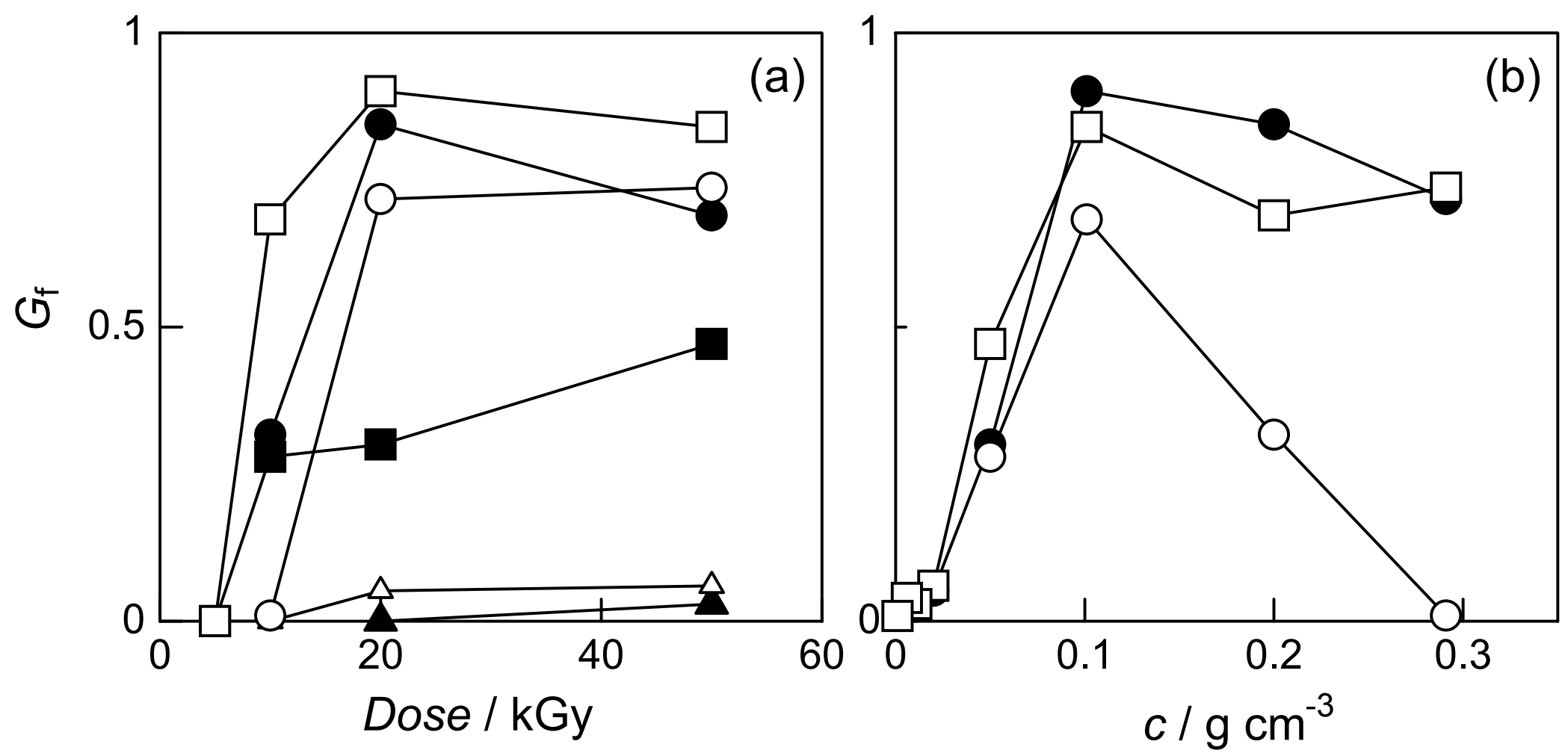

Figure 1. Terao et al. 


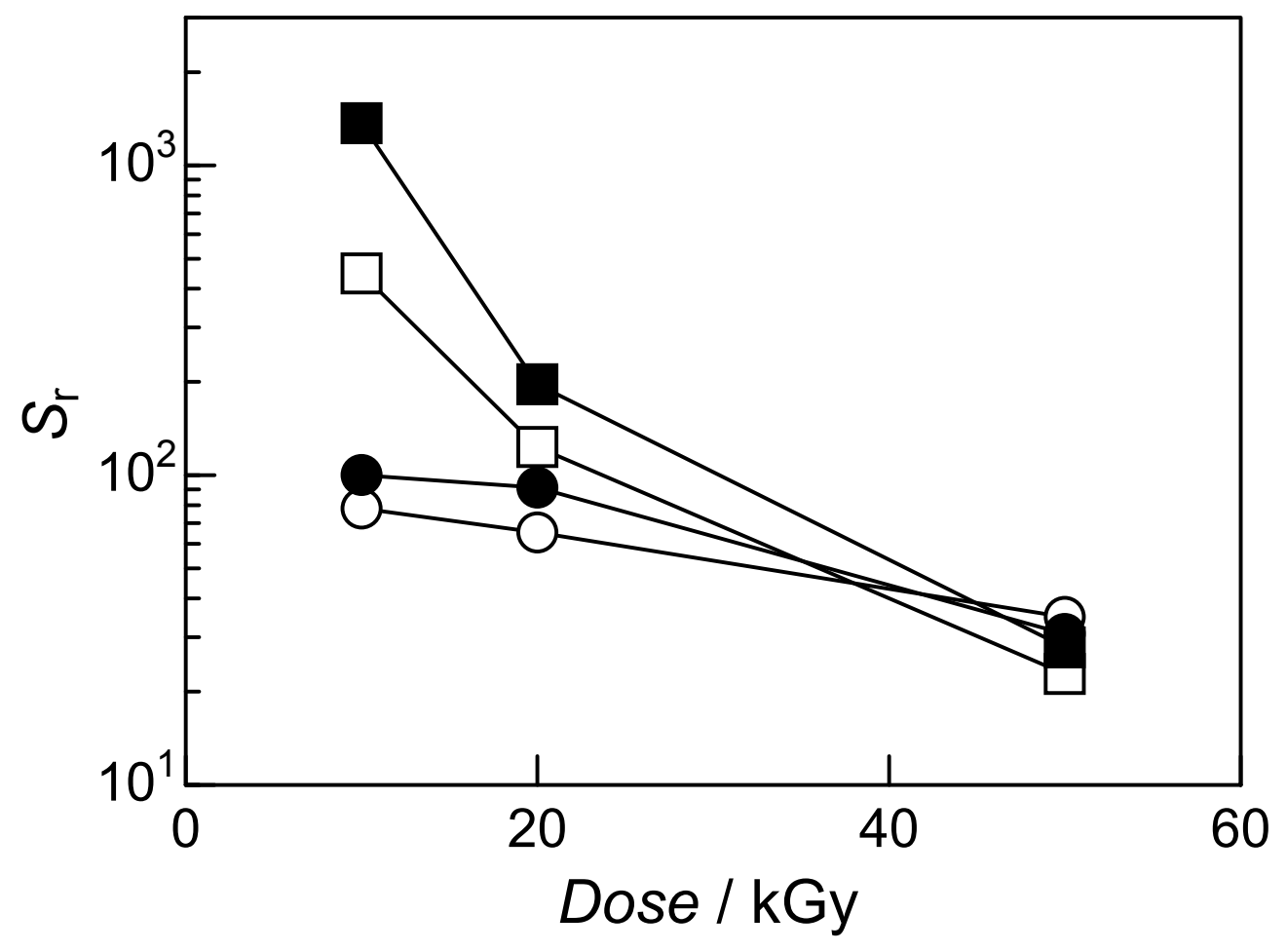

Figure 2. Terao et al. 

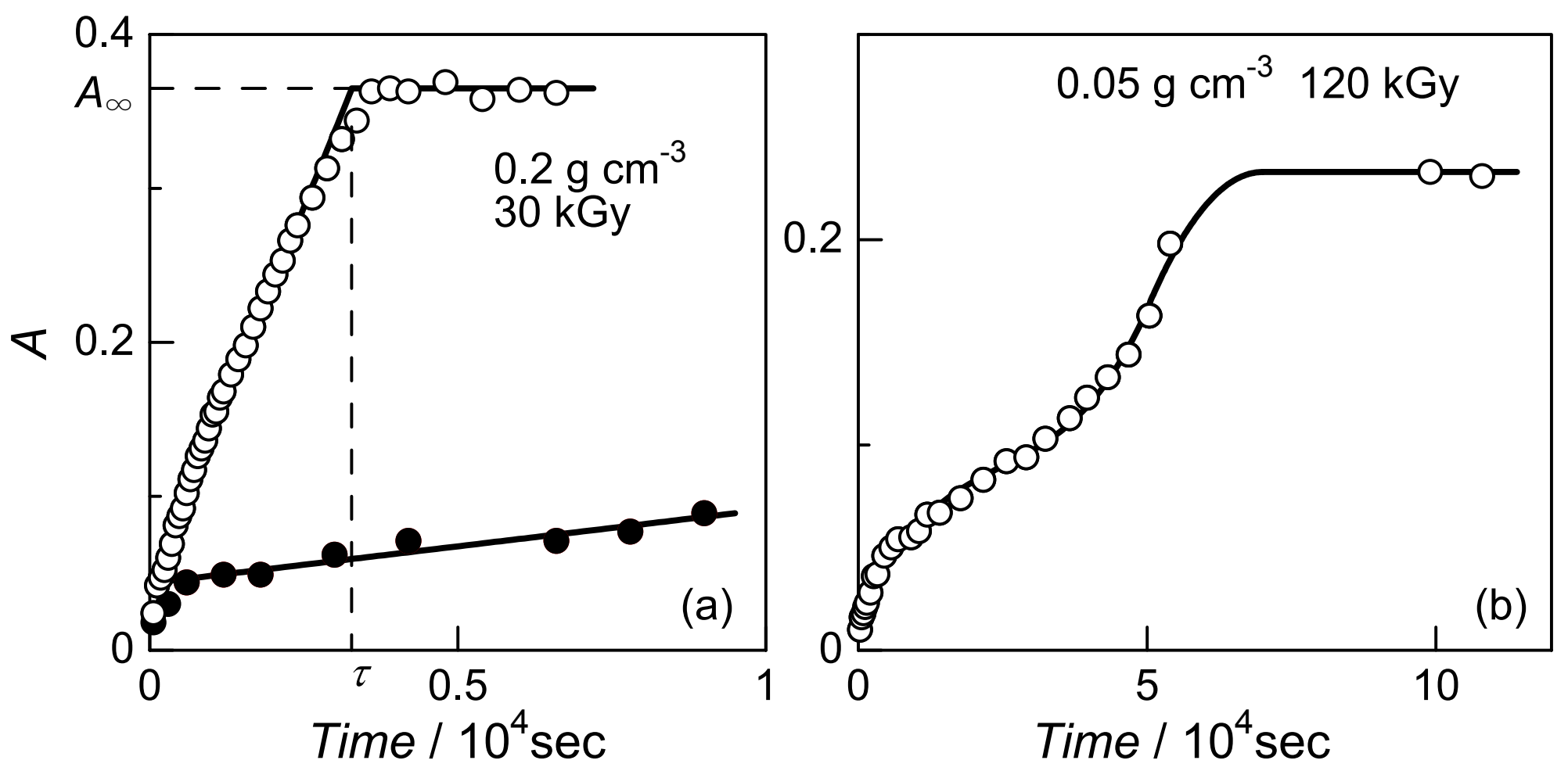

Figure 3. Terao et al. 

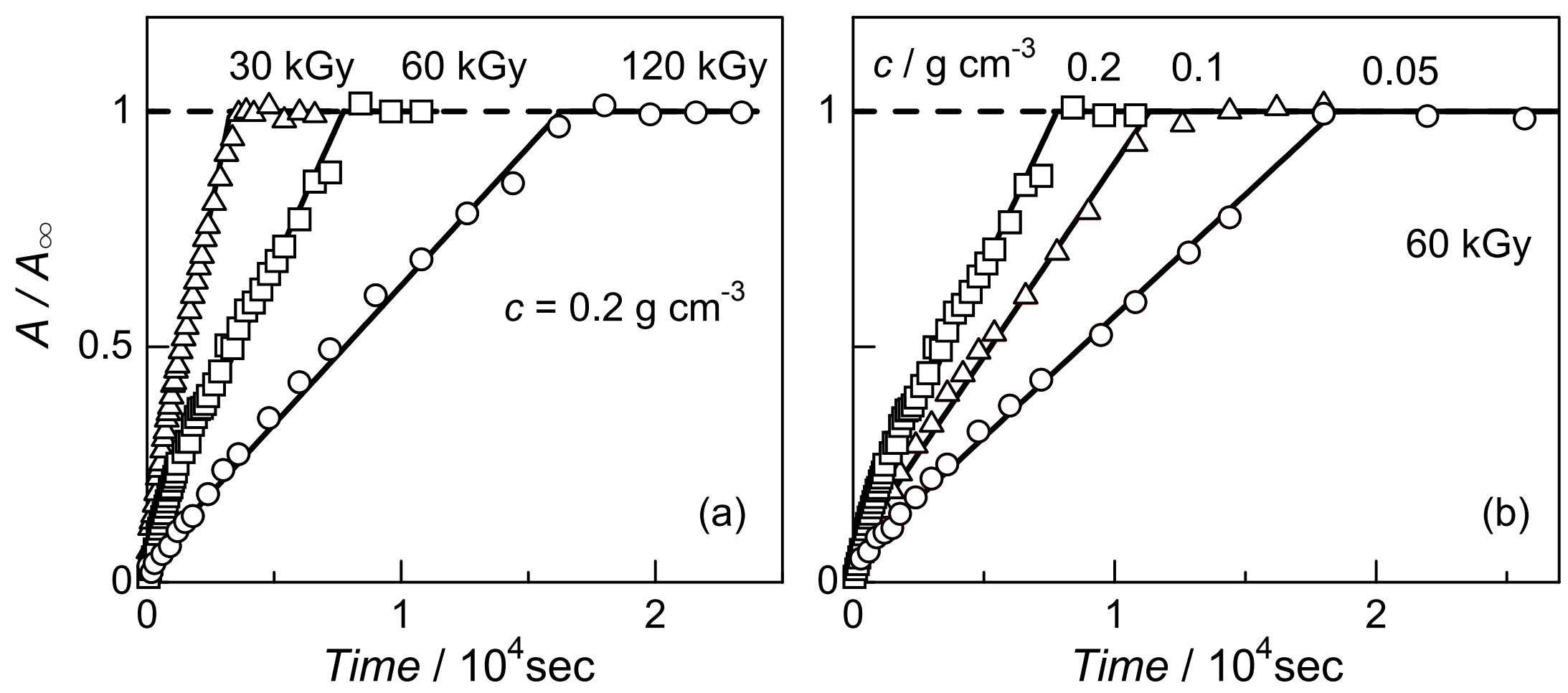

Figure 4. Terao et al. 


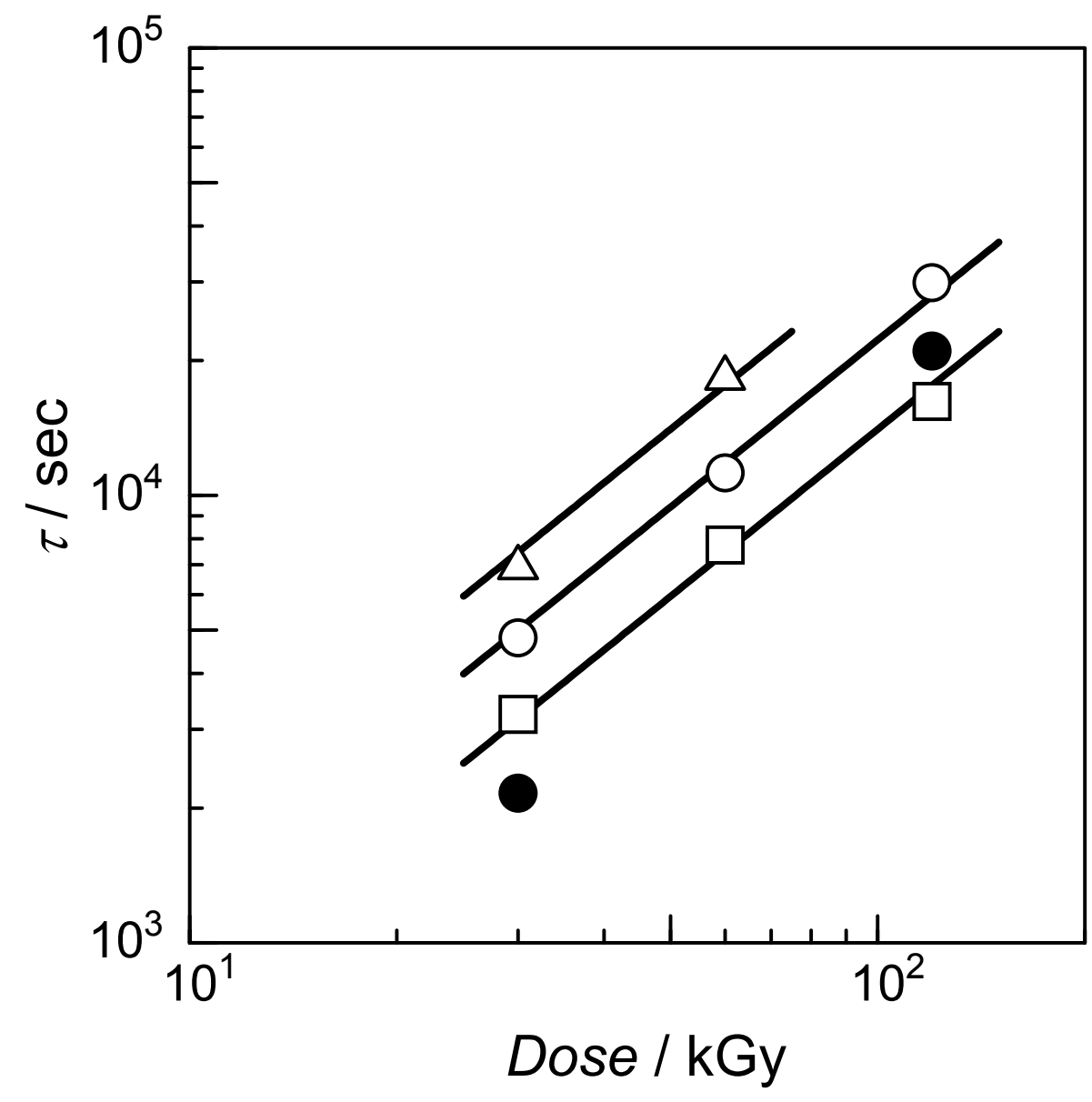

Figure 5. Terao et al. 


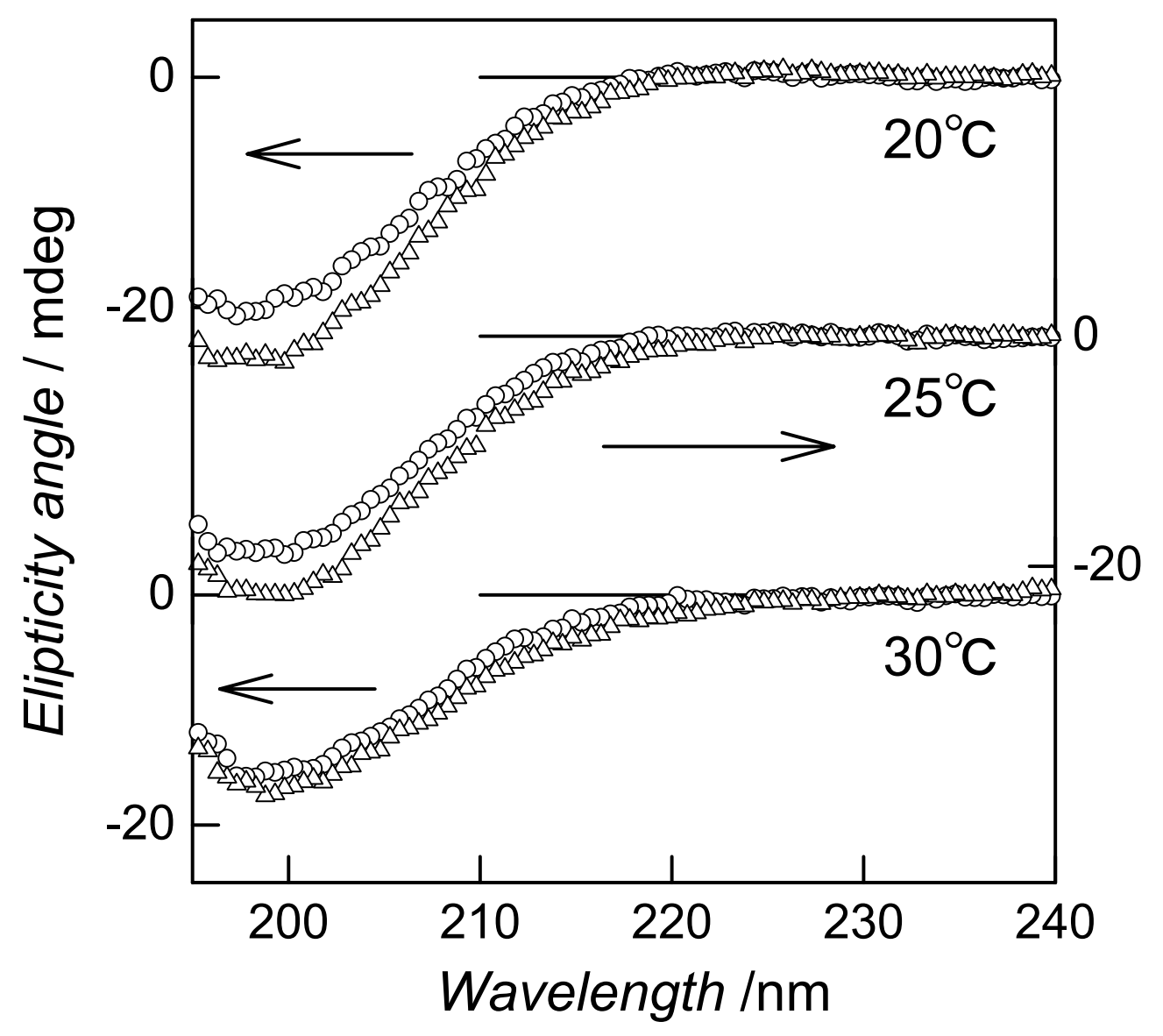

Figure 6. Terao et al. 


\section{Figure Caption}

Figure 1. Dose (a) and concentration (b) dependence of the gel fraction $G_{\mathrm{f}}$ of the irradiated aqueous gelatin from porcine skin (type A). In panel (a), unfilled circles, $0.29 \mathrm{~g} \mathrm{~cm}^{-3}$; filled circles, $0.20 \mathrm{~g} \mathrm{~cm}^{-3}$; unfilled squares, $0.10 \mathrm{~g} \mathrm{~cm}^{-3}$; filled squares, $0.050 \mathrm{~g} \mathrm{~cm}^{-3}$; unfilled triangles, $0.020 \mathrm{~g} \mathrm{~cm}^{-3}$; filled triangles, $0.010 \mathrm{~g} \mathrm{~cm}^{-3}$. In panel (b), unfilled circles, $10 \mathrm{kGy}$; filled circles, 20 kGy; unfilled squares, $50 \mathrm{kGy}$.

Figure 2. Dose dependence of the swelling ratio $S_{\mathrm{r}}$ of the irradiated aqueous gelatin from porcine skin (type A): unfilled circles, $0.29 \mathrm{~g} \mathrm{~cm}^{-3}$; filled circles, $0.20 \mathrm{~g} \mathrm{~cm}^{-3}$; unfilled squares, $0.10 \mathrm{~g} \mathrm{~cm}^{-3}$; filled squares, $0.050 \mathrm{~g} \mathrm{~cm}^{-3}$.

Figure 3. Time courses of the absorption $A$ at $275 \mathrm{~nm}$ of the buffer solution soaking gelatin hydrogel irradiated with electron beam. (a) For the $0.2 \mathrm{~g} \mathrm{~cm}^{-3} \mathrm{gel}(1 \mathrm{~mm}$ thickness) at $30 \mathrm{kGy}$. (b) For the $0.05 \mathrm{~g} \mathrm{~cm}^{-3}$ gel ( $1 \mathrm{~mm}$ thickness) at $120 \mathrm{kGy}$. Unfilled circles: with enzyme, filled circles: without enzyme.

Figure 4. Time courses of the reduced absorbance $A / A_{\infty}$ of the buffer solution. (a) For $0.2 \mathrm{~g}$ $\mathrm{cm}^{-3}$ gelatin solution at indicated dose. (b) For the gel irradiated with $60 \mathrm{kGy}$ at indicated gelatin concentration.

Figure 5. Plots of the decomposition time $\tau$ vs dose for $0.05 \mathrm{~g} \mathrm{~cm}^{-3}$ (triangles), $0.10 \mathrm{~g} \mathrm{~cm}^{-3}$ (circles) and $0.20 \mathrm{~g} \mathrm{~cm}^{-3}$ (squares), and unfilled and filled symbols for $1.0 \mathrm{~mm}$ and $0.5 \mathrm{~mm}$ thickness of the gel sheet.

Figure 6. Circular dichroism spectra of $1 \times 10^{-4} \mathrm{~g} \mathrm{~cm}^{-3}$ gelatin from porcine (triangles) and 
bovine (circles) in phosphate buffer $\mathrm{pH} 7.0$ at indicated temperatures. 